\title{
Rigorous Results for Aperiodic and Almost Periodic Substitution Sequences
}

\author{
S. T. R. Pinho \\ Instituto de Física \\ Universidade Federal da Bahia \\ 40210-340, Salvador, BA, Brazil \\ e-mail:suani@ufba.br \\ and T. C. Petit Lobão \\ Departamento de Ciências Exatas \\ Universidade Estadual de Feira de Santana \\ 44031-460, Feira de Santana, BA, Brazil \\ e-mail: thierry@uefs.br
}

Received on 15 August, 2000

\begin{abstract}
Recent studies of the effects of geometric fluctuations, associated with aperiodic exchange interactions, on the critical behavior of ferromagnetic Ising models on hierarchical lattices, were the motivation to investigate some properties of two-letter sequences generated by uniform substitutions. We rigorously identify the substitutions that generate either periodic or aperiodic (almost periodic or not) sequences. For instance, two known substitutions, the Thue-Morse and the perioddoubling rules, generate aperiodic sequences of almost periodic type.
\end{abstract}

\section{Introduction}

The aperiodic character of structures such as sequences generated by substitution rules defined on a finite alphabet has been investigated by several authors in recent years. In the realm of physics, this is related to the discovery, in the late 1980's, of new structures called quasicrystals [1]. There are several theoretical studies of aperiodic structures associated with infinite sequences generated by successive juxtapositions of letters of a finite alphabet according to some substitutional rule. For example, the Fibonacci rule,

$$
\rho:\left\{\begin{array}{l}
a \mapsto a b \\
b \mapsto a
\end{array}\right.
$$

generates an infinite sequence by successive applications of the rule on the letter $a$,

$$
\begin{gathered}
a \rightarrow \rho(a)=a b \rightarrow \rho^{2}(a)=\rho(a) \rho(b)= \\
=a b a \rightarrow \rho^{3}(a)=a b a a b \rightarrow \rho^{4}(a)=a b a a b a b a \ldots
\end{gathered}
$$

Although these substitutional sequences have been studied by mathematicians mainly, in the context of algebra [2] and dynamical systems [3], there is also a close link between substitutional sequences and physical systems [4]. For example, they have been used to construct aperiodic electronic systems and spin models whose exchange couplings $J_{i}$ are defined by a substitution rule. Consider a one-dimensional Ising model, with nearest-neighbor exchange interactions $J_{a}$ and $J_{b}$, according to the rule (1). In the thermodynamic limit, this "Fibonacci chain" corresponds to the $k^{t h}$ power of $\rho(a)$, from Eq. (2), for $k \rightarrow \infty$. It is certainly interesting to investigate the role of aperiodicity on the properties of these model systems.

The effects of geometric fluctuations on the critical behavior of spin models with aperiodic exchange interactions is an important topic of equilibrium statistical mechanics. There is a heuristic criterion, proposed by Luck [6], in analogy with the Harris criterion for ferromagnetic disordered systems [5], to account for the relevance of the geometric fluctuations. According to Luck's criterion, if the exchange interactions of a ferromagnetic Ising model obey a substitution rule, the critical behavior changes with respect to the pure periodic model (for $J_{a}=J_{b}$ ) if the fluctuations are unbounded. These fluctuations are related to the difference between the number of occurrences of a certain letter on a finite generation and the expected number if we consider the frequency in the infinite word [7].

Some recent publications [8] refer to a ferromagnetic Ising model on a generalized diamond hierarchi- 
cal lattice (DHL) [9]. The exchange interactions along the branches of the DHL lattice are chosen according to suitable binary (two-letter) substitutional rules (see Figure 1). Note that, to preserve the topology of the DHL (each branch has the same number of bonds $\ell$ ), the substitutions have to be uniform (each letter generates words of the same length $\ell$ ).

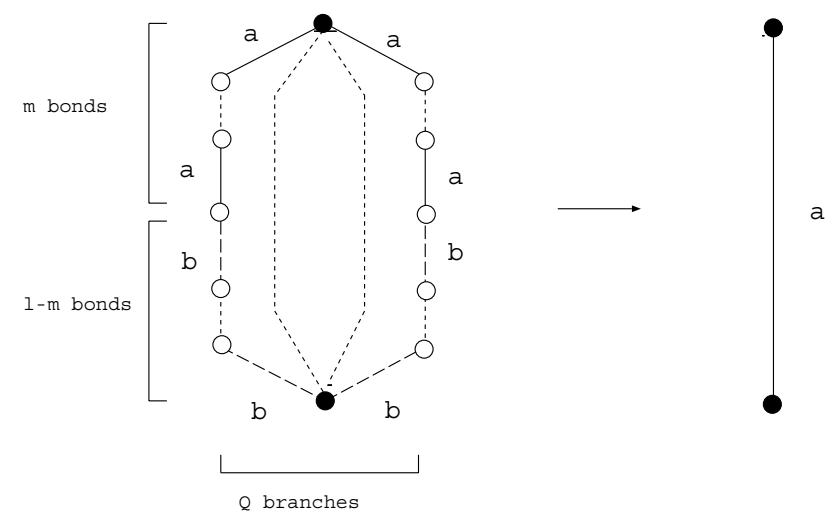

Figure 1. The deflation rule associated with a substitution (inflation) rule that transforms the coupling constant of type $a$ such that $a \mapsto a^{m} b^{\ell-m}$ in a generalized $(\ell$ bonds by branch) diamond hierarchical lattice (DHL). This rule is applied for each branch of the DHL.

Some of these uniform binary substitutions generate aperiodic sequences (which may or may not be almost periodic). Also, there are some of those substitutions that generate just periodic sequences. In this work, we identify classes of periodic, almost periodic, and aperiodic infinite sequences, associated with these substitutions, and present some rigorous results related to their periodic/aperiodic character. Most of these results may be extended to some classes of substitution with any number of letters. Section II presents the basic concepts and results of the theory. Our results and proofs related to periodicity, aperiodicity, and almost periodicity of the sequences are presented in Section III. Finally, in Section IV we present some concluding remarks and a discussion of some important aspects of these results for the analysis of spin models.

\section{Basic Concepts}

Most of the concepts presented in this Section can be found in the works of Queffelec [3] and Cobham [10].

Let $\mathcal{A}$ be a finite set, called the alphabet, whose elements, $x$, are letters. The juxtaposition of $\ell$ letters, not necessarily different, is called a finite word of length $\ell$. An infinite (right) word results from the juxtaposition of an infinite number of letters with an initial one (on the left). The set of all finite words with letters in $\mathcal{A}$ is called $\mathcal{A}^{*}$; we note by $\mathcal{A}^{\mathbf{N}}$ the set of all infinite words, since an element of $\mathcal{A}^{\mathbf{N}}$ is an application of the set of the natural numbers $\mathbf{N}$ in $\mathcal{A}$, i.e., a mathematical sequence. For $\mathcal{A}=\{a, b\}$, we have $\mathcal{A}^{*}=\{a, a b, a a, b b, a a b, a b b, \ldots\}$ and $\mathcal{A}^{\mathbf{N}}=$ $\{a b a a a \ldots, \quad a a b b a \ldots, \quad \ldots\}$

Definition II.1 $A$ substitution $\rho$ on an alphabet $\mathcal{A}$ is a map

$$
\begin{aligned}
\rho: \mathcal{A} & \rightarrow \mathcal{A}^{*} \\
x & \mapsto \rho(x),
\end{aligned}
$$

which associates with each letter $x$, in $\mathcal{A}$, a finite word $\rho(x)$ in $\mathcal{A}^{*}$.

Definition II.1.1 A substitution $\rho$ is binary if the number of letters of $\mathcal{A},|\mathcal{A}|$, is 2 .

Definition II.1.2 A substitution $\rho$ is uniform of modulus $\ell$ if, to every $x$ in $\mathcal{A}$, the number of letters in $\rho(x)$ is $\ell$, in symbols, $|\rho(x)|=\ell$.

Definition II.1.3 A uniform substitution $\rho$ is modular if $\rho(x)=X$, for all $x$ in A and $X$ is a finite word.

Definition II.1.4 A uniform binary substitution $\rho$ is alternated if $\rho(a)=a b a b \ldots a$ or $a b a b \ldots b$ and $\rho(b)=b a b a \ldots b \quad$ or $\quad b a b a \ldots a$, respectively.

The Fibonacci substitution (1) is binary but is not uniform. The Rudin-Shapiro substitution is uniform but is not binary, since it is defined on $\mathcal{A}=\{a, b, c, d\}$ by $\rho: a \mapsto a c, b \mapsto d c, c \mapsto a b, d \mapsto d b$. An example of a class of uniform binary substitutions of modulus $\ell$ is given by

$$
\begin{aligned}
\rho: \mathcal{A} & \rightarrow \mathcal{A}^{*} \\
a & \mapsto a^{m} b^{\ell-m}, \\
b & \mapsto b^{q} a^{\ell-q},
\end{aligned}
$$

where $\ell>1$ and $m \neq 0$ or $q \neq 0$. The Thue-Morse and period-doubling substitutions are particular cases of (4) with $\ell=2$, where $m=q=1$ and $m=1, q=0$, respectively.

It is obvious that a substitution $\rho: \mathcal{A} \rightarrow \mathcal{A}^{*}$ may be extended by concatenation to $\rho: \mathcal{A}^{*} \rightarrow \mathcal{A}^{*}$ and to $\rho: \mathcal{A}^{\mathbf{N}} \rightarrow \mathcal{A}^{\mathbf{N}}$. By concatenation we mean $\rho\left(a_{1} a_{2} \ldots a_{j}\right)=\rho\left(a_{1}\right) \rho\left(a_{2}\right) \ldots \rho\left(a_{j}\right)$ and $\rho\left(a_{1} a_{2} \ldots\right)=$ $\rho\left(a_{1}\right) \rho\left(a_{2}\right) \ldots$. By successive applications of a substitution $\rho$, starting, for instance, from letter $a$, it is generated a sequence of finite words $\left(a, \rho(a), \rho^{2}(a), \ldots\right)$; we may observe this process for the Fibonacci substitution in (2).

Definition II.2 The substitution matrix $\mathcal{M}$ associated with the substitution $\rho: \mathcal{A} \rightarrow \mathcal{A}^{*}$ is an $n \times n m a-$ trix, where $n=|\mathcal{A}|$, whose elements $m_{i j}$ are the number of occurrences of letter $x_{i}$ in $\rho\left(x_{j}\right)$, in symbols $m_{i j}=\left|\rho\left(x_{j}\right)\right|_{x_{i}}$. 
For the class of substitutions (4), we have

$$
\mathcal{M}=\left(\begin{array}{cc}
m & \ell-q \\
\ell-m & q
\end{array}\right)
$$

whose eigenvalues are the integer values: $\lambda_{1}=\ell$ and $\lambda_{2}=m+q-\ell$. We may observe that substitutions $\rho$, such that $a \mapsto a Y, b \mapsto W$ with $|Y|_{a}=m-1$, $|Y|_{b}=\ell-(m-1),|W|_{b}=q$ and $|W|_{a}=\ell-q$ have the same associated matrix $\mathcal{M}$. For example, the modular binary substitution ( $a \mapsto a b, b \mapsto a b)$ has the same matrix of Thue-Morse substitution whose eigenvalues are $\lambda_{1}=2$ and $\lambda_{2}=0$.

It is easy to show that the eigenvalues of the associated matrix of any modular binary substitution of modulus $\ell$ are $\lambda_{1}=\ell$ and $\lambda_{2}=0$, since the matrix elements are such that $m_{11}=m_{12}$ and $m_{21}=m_{22}$. Note that, even in case $|X|_{b}=0$ (or $|X|_{a}=0$ ), we have $\lambda_{1}=\ell$ and $\lambda_{2}=0$.

Analogously, the eigenvalues of the associated matrix of an alternated substitution of odd modulus $\ell$ are $\lambda_{1}=\ell$ and $\lambda_{2}=1$, since the matrix elements are such that $m_{11}=m_{22}$ and $m_{12}=m_{21}=m_{11}-1$.

Definition II.2.1 A substitution matrix $\mathcal{M}$ is primitive if there exists a positive integer $r$ such that all elements of $\mathcal{M}^{r}$ are strictly positive.

Definition II.3 Let $\rho$ be a substitution; an element $\chi$ of $\mathcal{A}^{\mathbf{N}}$ is a substitution sequence of $\rho$ if $\chi$ is a fixed point of $\rho, i$. e., $\rho(\chi)=\chi$.

Remark I If $\chi$ is a substitution sequence, it is easy to demonstrate that

$$
\rho\left(\chi_{1}\right)=\chi_{1} Y
$$

where $\chi_{1}$ means the first letter of $\chi$ and $Y$ is a finite word.

Reciprocally, for successive applications of $\rho$ starting, for instance, from $a$, if the first letter of $\rho(a)$ is $a$, that is, $\left.\rho(a)\right|_{1}=a$, then $\rho^{k+1}(a)=\rho^{k}(a) W$, for all $k$, where $W$ is a finite word (see (2) for a Fibonacci rule). In this sense, we say that the sequence of powers $\rho^{k}(a)$ is self-similar. This property guarantees, in case $|\rho(a)|>1$, that the substitution $\rho$ determines a substitution sequence $\chi$ that may be denoted by the symbol

$$
\chi=\lim _{k \rightarrow \infty} \rho^{k}(a)
$$

and, obviously, $\chi_{1}=a$.

Definition II.3.1 A substitution sequence is periodic with no radical if it is generated by infinite successive occurrences of a block $T$ (which is called period) with length $t=|T|(\chi=T T T T T \ldots)$. If the periodic substitution sequence $\chi$ has a radical $R$, it may be expressed by $\chi=R T T T \ldots$ A substitution is aperiodic if it is non-periodic.

Definition II.3.2 A substitution sequence is almost periodic if, for all block (finite word) $Y$, occurring in $\chi$, there exists, at least, a positive integer $n$ such that $Y$ occurs in each word of length $n$ contained in $\chi$.

In other words, the distance between any two successive occurrences of any block is limited. For a periodic sequence, this distance is zero; so, we may say that, in a certain sense, periodic sequences are almost periodic as well.

Theorem II.1 Let be a uniform substitution $\rho$ that generates a substitution sequence $\chi$. If its substitution matrix $\mathcal{M}$ is primitive, so $\chi$ is almost periodic.

The demonstration of this theorem is presented in reference [10]. This result may be generalized for a nonuniform substitution [11].

\section{Results}

In this Section, we analyze substitutions $\rho$ that are binary $(\mathcal{A}=\{a, b\})$, uniform $(|\rho(a)|=|\rho(b)|)$, and determine a substitution sequence $\chi$, i. e., $\rho(\chi)=\chi$; thus the first letter, $\chi_{1}$, in the sequence $\chi$, is such that $\rho\left(\chi_{1}\right)=\chi_{1} Y$ (see Remark I). We now assume that this first letter is $a$. Therefore, we write

$$
\begin{aligned}
\rho: \mathcal{A} & \rightarrow \mathcal{A}^{*} \\
a & \mapsto a Y, \\
b & \mapsto W,
\end{aligned}
$$

with $|Y|=\ell-1,|W|=\ell$ and $\ell>1$.

Most of the results of this section are based on the following general definition:

DEFINITION III.1 Let $\chi \in \mathcal{A}^{\mathbf{N}}, \chi=\chi_{1} \chi_{2} \ldots$; the letter $a_{k} \in \mathcal{A}=\left\{a_{1}, a_{2}, \ldots\right\}$ is co-final in $\chi$ if, for any natural number $r$, there exists a natural number $s$, greater than $r$, such that $a_{k}=\chi_{s}$. We write $a_{k} \in \infty$.

We may notice that, if a letter $a_{k}$ is co-final in $\chi$, it appears infinitely often in $\chi$.

\section{III.1 Periodicity}

We have two trivial periodic substitution sequences determined by (6), that will be called of simple period; these are related to the pure case of the spin model. Let us prove the following theorem.

THEOREM III.1 If one of the two letters of the alphabet $\mathcal{A}=\{a, b\}$ is not co-final in $\chi$, then the sequence 
$\chi$ is periodic, with period $T$ such that $|T|=1$, and it presents only two types:

(I) $\chi=a a a a . .$. , if $b$ is not co-final in $\chi$;

(II) $\chi=a b b b \ldots$, if $a$ is not co-final in $\chi$.

Demonstration: Let us suppose that $b \notin \infty$ : since $\chi$ is infinite, then $a \in \infty$; if $b \in Y$, inasmuch as $\rho(a)=a Y$, then $b \in \infty$. So, we conclude that $b \notin Y$; since $\chi=\lim _{k \rightarrow \infty} \rho^{k}(a)$, it follows that $\chi=a a a \ldots$.

Now, let us suppose that $a \notin \infty$. Then, analogously, $b \in \infty$. If $a \in W$, then $a \in \infty$, because $\rho(b)=W$; so $a \notin W$. If $a \in Y$, since $\rho(a)=a Y, \rho^{2}(a)=a Y \rho(Y)$, and so on, we would have $a \in \infty$. So, we conclude that $a \notin Y$, and it is easy to see that $\chi=a b b b b \ldots$

The next theorem is a cornerstone to many results that follow.

THEOREM III.2 Let $\chi$ be a periodic substitution sequence; the first letter $a$ is co-final in $\chi$ if, and only if, $\chi$ does not have radical.

Demonstration: Suppose that $\chi=R T T T T \ldots$ and $|T|=t$. Since $a$ is co-final in $\chi$ and $\chi$ is periodic, then $a \in T$. Take $a \in T$ for a fixed block $T$; it is possible to consider a finite number $n$ of iterations such that the block generated from $a$ is $\rho^{n}(a)=R T X$ where $X$ is a finite word. But $\rho^{n}(a)$ is also in the periodic part of $\chi$; so $R$ also occurs in the periodic part of $\chi$. If we count $t=\left|T^{\prime}\right|$ letters from the first $a$, we have $\chi=T^{\prime} T^{\prime} T^{\prime} T^{\prime} \ldots$, in other words, $\chi$ does not have a radical.

For the converse, if $\chi$ does not have radical, it is periodic from the first letter $a$ of the sequence and it is obvious that $a$ is co-final in $\chi$ since $\chi=T T T T \ldots$.

In view of the last results, we will always assume that the substitution $\rho$ in (6) is such that the number of letters $b$ in $\rho(a)$ is non-zero, or, in symbols $|\rho(a)|_{b}>0$. Moreover, we may also assume that both letters $a$ and $b$ are co-final in $\chi$.

LEMMA III.1 Consider a substitution sequence $\chi$ generated by the substitution $\rho$, where both letters are co-final in $\chi$. If $\rho$ is modular, then $\chi$ is periodic without radical and the length of the period, $|T|$, is equal or smaller than the modulus $\ell$. Conversely, if $\chi$ is periodic without radical and such that $|T|=\ell$, then $\rho$ is a modular substitution.

Demonstration: If $\rho: a \mapsto X$ and $b \mapsto X$, then $\rho^{2}(a)=\rho(X)=X \ldots X$ with $\left|\rho^{2}(a)\right|=\ell^{2}$. By finite induction, since $\chi=\lim _{k \rightarrow \infty} \rho^{k}(a)$, we conclude that $\chi=X X X X \ldots$; obviously, $|T| \leq|X|$.
For the converse, if $\chi$ is periodic with no radical and $|T|=\ell$, then $\rho(a)=T$. Since both letters are co-final in $\chi$, they belong to the period and $\rho^{2}(a)=\rho(T)=T \ldots T$. So, we also have $\rho(b)=T$; therefore, the substitution is modular.

LEMMA III.2 If the modulus $\ell$ of an alternated $s u b$ stitution $\rho$ is odd, then the substitution sequence is periodic with period $T=a b$.

Demonstration: Since $\ell$ is odd, $\rho: a \mapsto a b \ldots a$, $b \mapsto b a \ldots b$. By concatenation,

$\rho^{2}(a)=\rho(a) \rho(b) \ldots \rho(a)=a b \ldots a b a \ldots b \ldots \ldots a b \ldots a$,

and so on. Thus, by induction, we have, for any $k$, $\rho^{k}(a)=T T \ldots T T$, with $T=a b$. Therefore, the substitution sequence is periodic with $T=a b$.

\section{III.2 Almost periodicity}

We divide the substitutions described in (6) into two classes:

(a) $|W|_{a}=0$,

(b) $|W|_{a}>0$.

LEMMA III.3 Sequences associated with substitutions of type (a) are not almost periodic.

Demonstration: Since $\left|\rho^{k}(b)\right|=k \ell$ for any $k$, it is easy to see that there are arbitrarily large blocks in $\chi$ without the letter $a$. So the block $Y=a$ may be arbitrarily far from another such block. In other words, the substitution sequence is not almost periodic.

LEMMA III.4 Sequences associated with substitutions of type (b) are almost periodic.

Demonstration: It is easy to prove that the substitution matrix is primitive. Let $Y$ and $W$ such that $|Y|_{a}=m-1,|Y|_{b}=\ell-(m-1),|W|_{b}=q$ and $|W|_{a}=\ell-q$. Since $\ell>q$ (even when $\left.q=0\right)$, the matrix (5) is primitive because both elements of the second diagonal, $m_{21}=\ell-m$ and $m_{12}=\ell-q$, are non-zero. Therefore, for any positive integer $r$, all elements of $\mathcal{M}^{r}$ are strictly positive. Using Theorem II.1, it is proved that the sequence is almost periodic.

Let us generalize LEMMA III.4 for non-uniform binary substitutions whose eigenvalues are irrational numbers. For this purpose, consider the substitution matrix such that $a \mapsto a Y$ and $b \mapsto W$, with $|Y|_{a}=$ 
$m-1,|Y|_{b}=n,|W|_{a}=p$ and $|W|_{b}=q$; if its eigenvalues are irrational numbers, it is easy to prove that $p \neq 0$ and $n \neq 0$. By an argument analogous to the previous demonstration, $\mathcal{M}$ is primitive. Using the generalization of Theorem II.1 for non-uniform substitutions, the sequence is also almost periodic. These sequences generated by substitutions with irrational eigenvalues (as, for example, the Fibonacci substitution) may be called quasiperiodic since they have a strong relation to the quasicrystals.

\section{Aperiodicity}

Using the previous results, we obtain the following theorems about aperiodicity:

THEOREM III.3 Sequences associated with substitutions (6) such that $W=b^{\ell}\left(|W|_{a}=0\right)$ are aperiodic.

Demonstration: Since $a \in Y$ and $b \in Y, a$ and $b$ are co-final in $\chi$. Suppose that the substitution sequence is periodic; using THEOREM III.2, the sequence has no radical. Since $W=b^{\ell}$, there are blocks of $b$ arbitrarily large. However, the period is finite and $\chi=T T T T \ldots$, so $T$ is just formed by $b$ 's. Therefore, there is a contradiction because there exist $a$ 's in $\chi$.

THEOREM III.4 Sequences associated with substitutions (6) such that $Y=X b$ and $W=Z a$, with $X$ and $Z \in \mathcal{A}^{*}$, are aperiodic.

Demonstration: Since $\mathcal{A}$ is finite and the sequence $\chi$ is infinite, some letter is co-final in $\chi$. If this letter is $a$, the letter $b$ is also co-final in $\chi$ because $a \mapsto a X b$. Analogously, for $b$, the letter $a$ is also co-final in $\chi$, since $b \mapsto Z a$. So, the letters $a$ and $b$ are co-final in $\chi$. Suppose that the sequence $\chi$ is periodic. Using THEOREM III.2, inasmuch as both letters are co-final in $\chi$, the sequence is $\chi=T T T T \ldots$, where $T$ is the period. Since the substitution is uniform of length $\ell$, the last letter of the period $T$, in a $k$-iteration, must be the same as the last letter of the $\ell$ periods $T$ generated from the period $T$ in the $(k+1)$-iteration. However, for this class of substitutions, $\rho(a)=a X b$ and $\rho(b)=Z a$. So, we have a contradiction; thus, the sequence is aperiodic.

\section{Discussion and Conclusions}

For sequences generated by binary substitutions, the geometric fluctuations are related to the smallest eigenvalue $\lambda_{2}$ of the substitution matrix. For any substitution which generates a simple periodic sequence with $|T|=1$, we have $\lambda_{2}=0$ (this is a particular case of a modular substitution as discussed in Section II). This means that there are no geometric fluctuations in the pure model. Even for modular substitutions (whose words $\rho(x)$ have the letters $a$ and $b$ ), $\lambda_{2}=0$ (see Section II), and the substitution sequence is periodic with $T=\rho(a)=\rho(b)$ (see LEMMA III.1 in Section III). In this sense, in view of Luck's criterion, the non-pure periodic models (for $J_{a} \neq J_{b}$ ), with $|T|>1$, are similar to the pure models (for $J_{a}=J_{b}$ ). Also, the aperiodic models, generated by substitutions with the same matrix as a modular one, present irrelevant geometrical fluctuations.

For alternated substitutions of odd modulus, we have seen in Section II that $\lambda_{2}=1$. In LEMMA III.2, we proved that these substitution sequences are periodic. For one-dimensional ferromagnetic Ising models whose couplings obey these substitution rules, this corresponds to a marginal case $\left(\lambda_{2}=1\right)$, according to Luck's criterion. However, for the Ising model on the generalized DHL, we may prove (see reference [12]) that the geometrical fluctuations are also irrelevant. This is also the case for aperiodic substitution sequences with the same matrix as an alternated one of odd modulus.

Relevant fluctuations only occur for aperiodic models. In other words, aperiodicity is a necessary condition for changing the critical behavior. It is then necessary to prove the aperiodic character of the substitution sequences. However, aperiodicity is not a sufficient condition. If the fluctuations are bounded, the aperiodic model belongs to the same universality class as the correspondent pure model.

Analogously, for almost periodic sequences, the fluctuations may be relevant or irrelevant. However, for a one-dimensional Ising model whose couplings are chosen according to a uniform binary substitution, it is easy to see that, if the sequence is not almost periodic $(\ell=q)$, the fluctuations are always relevant, because $\lambda_{2}=m>1$. It is interesting to point out that, for the Ising model in the generalized DHL, this condition does not guarantee the relevance of fluctuations.

In reference [8], two examples of aperiodic and also almost periodic models on hierarchical lattices are presented. While the fluctuations are relevant for one of them, they are irrelevant for the other one. The relevance of the fluctuations depends on the substitution rule (through the eigenvalues of the substitution matrix), on the topological characteristics of the lattice (the results may differ for a DHL and a one-dimensional lattice) and also on the model (see references [7], [14] for the analyses of the Potts model on a DHL).

In this work, we present some results, with rigorous proofs, for uniform binary substitutions used for generating aperiodic exchange couplings of ferromagnetic spin models on a generalized DHL. Most of our results are based on THEOREM III.2, which is the cornerstone of our work. We prove the periodic character of the sequence generated by modular substitutions (LEMMA III.1) and by alternated substitutions of odd modulus (LEMMA III.2). We obtain a classification for almost 
periodic sequences (LEMMAS III.3 and III.4). We also demonstrate the aperiodic character of the substitution sequence associated with two classes of substitutions (THEOREMS III.3 and III.4). Most of the concepts and results of Section II are valid for alphabets with any finite number of letters. We conjecture that most of our results might be generalized for substitutions of any number of letters.

Acknowledgments: We thank S. R. Salinas for profitable questions that were the motivation of this work. We also thank him for a careful reading of this manuscript. We acknowledge several discussions with T. A. S. Haddad and S. R. Salinas. The first author acknowledges a fellowship from the program CAPES/PICD and the hospitality of Instituto de Física of Universidade de São Paulo, where part of this work was developed.

\section{References}

[1] D. Shechtman, I. Blech, D. Gratias, and J. W. Cahn, Phys. Rev. Lett. 53, 1951 (1984); D. Levine, and P. J. Steinhardt, Phys. Rev. Lett. 53, 2477 (1984).

[2] M. Lothaire, Combinatory on words in Encyclopedia of Mathematics and its applications 17, edited by G. Rota, Addison-Wesley Publishing Company, Massachusetts, 1983.

[3] M. Queffélec, Substitutional dynamics systems - Spectral Analysis, Lectures Notes in Mathematics 1294, SpringerVerlag, Berlin, 1987.
[4] J. M. Luck, P. Moussa, and M. Waldschmidt (editors), Number Theory and Physics, Springer Verlag, Berlin (1989).

[5] A. B. Harris, J. Phys. C 7, 1671 (1974).

[6] J. M. Luck, Europhys. Lett. 24, 359 (1993).

[7] T. A. S. Haddad, Comportamento crítico universal em ferromagnetos de Potts com interações aperiódicas, Master Dissertation, Universidade de São Paulo, São Paulo, 1999.

[8] S. T. R. Pinho, T. A. S. Haddad, and S. R. Salinas, Braz. J. Phys. 27, 567 (1997); S. T. R. Pinho, T. A. S. Haddad, and S. R. Salinas, Physica A, 257, 515 (1998).

[9] M. Kaufman and R. B. Griffiths, Phys. Rev. B 24, 496 (1981); R. B. Griffiths and M. Kaufman, Phys. Rev. B 26, 5022 (1982).

[10] A. Cobham, Math. Systems Theory 6, 164 (1972).

[11] O. B. R. Lovón, Medida de correlação quântica com potenciais de substituição de Thue-Morse, Master Dissertation, Universidade Federal de São Carlos, São Carlos, 1997.

[12] S. T. R. Pinho, Modelo de Ising em redes aperiódicas e criticalidade auto-organizada, $\mathrm{PhD}$ Thesis, Universidade de São Paulo, São Paulo, 1998.

[13] T. A. S. Haddad, S. T. R. Pinho, and S. R. Salinas, Phys. Rev. E 61, 3330 (2000).

[14] A. C. N. de Magalhães, S. R. Salinas, and C. Tsallis, J. Phys. A 31, L567 (1998). 